\title{
Factores asociados a la infección de vías urinarias en gestantes Pitalito, 2012
}

\section{Factors associated with urinary tract infection in pregnant women Pitalito, 2012}

\author{
María Torrejano ${ }^{1}$, Luz Calderón ${ }^{1}$, Ana Quimbayo $^{1}$ \\ 1. Enfermera Profesional Universidad Surcolombiana, Neiva, Huila \\ Programa de Epidemiologia USCO, Neiva, Huila \\ Correspondencia: María Fernanda Torrejano Delgado, Correo electrónico: mariafernandatorrejano- \\ delgado@gmail.com
}

\section{Resumen}

La infección de vías urinarias (IVU) es una patología prevalente durante la gestación, que de no ser intervenida a tiempo, presenta complicaciones como parto prematuro y bajo peso al nacer. Se realizó un estudio analítico tipo casos y controles, con el objetivo de identificar los factores asociados a la infección de vías urinarias en gestantes de la ESE Manuel Castro Tovar de la ciudad de Pitalito en el año 2012.

Se incluyeron 90 gestantes (45 casos y 45 controles) emparejadas por edad con diagnóstico de IVU, quienes asistieron a control prenatal. Se encontró relación epidemiológica entre la presencia de IVU en el embarazo y el "bajo" nivel educativo (OR: 1,33 IC 0.51-3.58), así como el antecedente de gestación (OR: 1,8 IC 0.54-6.83), control prenatal tardío (OR: 1,7 IC 0.55-5.58) y paridad (OR: 1,67 IC 0.55-5.58).

No se halló asociación entre la presencia de IVU en el embarazo con antecedente de IVU y ocupación; así mismo, un análisis con las variables propuestas en la hipótesis mostró asociación epidemiológica entre IVU en la gestación y los factores: bajo nivel de escolaridad y multiparidad, aunque esta diferencia no es estadísticamente significativa. Dentro de las limitaciones del estudio se encuentra el tamaño de la muestra y la calidad de las historias clínicas.

En conclusión, la presencia de IVU en el embarazo está sujeta a múltiples factores que van más allá del aspecto biológico, que deben tomarse en cuenta para su enfoque integral, optimizando el bienestar del binomio madre e hijo.

Palabras clave: Infección urinaria, embarazo, factor asociado. 


\begin{abstract}
Urinary tract infection (UTI) is a common pathology during pregnancy that if not treated on time, develops complications like preterm delivery and low weight birth.

An analytical case-control study was conducted aimed and identifying factors associated with urinary tract infection in pregnant women at ESE Manuel Castro Tovar from Pitalito in 2012.

Sample consisted of 90 pregnant women ( 45 cases and 45 controls) coupled by age with UTI diagnosis, who attended antenatal care. It was found epidemiological relation between UTI presence in pregnancy and "low" educational level (OR: 1,33 IC 0.51-3.58), as well as gestation antecedent (OR: 1,8 IC 0.54-6.83), late prenatal care (OR: 1,7 IC 0.55-5.58) and birth giving (OR: 1,67 IC 0.55-5.58).

There was no association between UTI presence in pregnancy with UTI antecedent and occupation; likewise, an analysis made with hypothesis variables showed epidemiological association between UTI in gestation and low educational level and multiple birth-giving factors, although this difference is not statistically significant. Sample size and medical histories quality are within the study limitations.

In conclusion, UTI presence during pregnancy is subject to multiple factors beyond the biological aspect which must be taken into consideration for its comprehensive approach, optimizing mother and child's welfare.
\end{abstract}

Keywords: urinary infection, pregnancy, associated risk

\section{Introducción}

La infección de vías urinarias (IVU) se considera como la invasión de los tejidos que hacen parte del aparato urinario por microorganismos patógenos. Es una de las patologías más prevalentes en la gestación ${ }^{(1,2,3)}$ durante la cual se presentan cambios anatómicos y fisiológicos importantes que contribuyen a su aparición ${ }^{(4,5)}$; así mismo, se presentan complicaciones para el binomio madre e hijo como el parto prematuro y el bajo peso al nacer ${ }^{(6,7)}$, en donde se determina que gestantes con infección urinaria "tienen 3 veces más posibilidades de tener un neonato con bajo peso al nacer" ${ }^{\prime(8)}$.

A nivel internacional se evidencia que si el embarazo es una condición de riesgo en las mujeres para adquirir alguna IVU ${ }^{(9)}$; características como la mayoría de edad, multiparidad, bajo nivel socioeconómico aumenta aún más dicho riesgo así como partos prematuros, bajo peso o mortalidad en el recién nacido.

En el departamento del Huila la situación no es diferente; aunque el establecimiento de los factores de riesgo para la presencia de IVU en las gestantes es precario debido al enfoque en las complicaciones; se observa que del 5 al 10\% de los partos pre-términos presentan una infección extrauterina por lo general en el tracto urinario aumentando dos o tres veces el riesgo de parto pre-termino $^{(10)}$.

Así mismo del 5,6\% de las gestantes que presentan IVU, el 2.1\% está asociada a bacteriuria asintomática, el $2 \%$ a cistitis aguda y el 1,4\% a pielonefritis aguda ${ }^{(11)}$ También se establece como factor de riesgo el nivel socioeconómico y, que la edad no es un factor de riesgo de importancia para presentar complicaciones asociadas a la $\operatorname{IVU}^{(12,13)}$

En Salud Pública, la Salud Materno-Infantil es el pilar del progreso social de una comunidad así como lo indicó la UNICEF en el 2010: “El resultado de las políticas sociales dirigidas a la primera infancia condiciona, en gran medida, el éxito o fracaso de las otras políticas sociales y las intervenciones que logran buenos resultados, además conllevan notorios beneficios en educación, en salud y en convivencia social durante la vida adulta. Por ello se dice que invertir en la primera infancia tiene un muy alto rendimiento 
económico y social”(14). Teniendo en cuenta que esa inversión abarca desde la etapa gestacional, es primordial enfocar todo tipo de acciones que prevengan la morbi-mortalidad del binomio madre-hijo.

Por lo anterior la presencia de complicaciones secundarias a la infección de la vía urinaria afecta el cumplimiento de políticas y metas trazadas por el gobierno nacional que busca mejorar la cobertura, calidad de la atención institucional de la gestación, parto, puerperio y fortalecer las acciones de vigilancia, prevención y atención de la mortalidad perinatal ${ }^{(15)}$.

El siguiente estudio buscó identificar factores socioeconómicos y gineco-obstétricos asociados a la infección de vías urinarias en gestantes de la ESE Manuel Castro Tovar para brindar recomendaciones sobre su prevención.

\section{Materiales y métodos}

Se realizó un estudio analítico de tipo casos y controles hospitalarios ${ }^{(16)}$ con gestantes de la ESE Manuel Castro Tovar que ingresaron al control prenatal entre enero y junio de 2012 con diagnóstico de IVU. Se aplicó el diseño de casos y controles hospitalarios con relación 1:1. Se definió caso como aquella gestante de la ESE Manuel Castro Tovar con infección de vía urinaria, que no presenta ninguna otra patología; los controles fueron las gestantes de la ESE Manuel Castro Tovar que no presentan infección de vías urinarias y ningún otro tipo de patología. Se calculó una muestra de 48 casos y 48 controles mediante OpenEpi, de los cuales aplicaron solo 45 casos (cumplían criterios de inclusión). Los controles se seleccionaron al azar en relación 1:1 con los casos según edad.

Se aplicó un cuestionario a las historias clínicas. Para completar la variable socioeconómica se retomó información del SISBEN (Sistema de Identificación y Clasificación de Potenciales Beneficiarios para los Programas Sociales). La confiabilidad del instrumento se realizó mediante prueba piloto con el $10 \%$ de historias clínicas escogidas al azar. La validez del mismo se efectuó por parte de profesionales en salud especializados en el área (Ginecólogo y epidemiólogo). Posteriormente se realizó la operacionalización de las variables utilizadas (Tabla 1 ).

Tabla 1. Operacionalización de las variables

\begin{tabular}{|c|c|c|}
\hline Variable & Definición & Nivel de medición \\
\hline Lugar de residencia & Sitio donde vive la madre & Nominal \\
\hline Edad & Tiempo que transcurre desde el nacimiento hasta la fecha & Razón \\
\hline Escolaridad & $\begin{array}{l}\text { Formación académica certificada por una Institución } \\
\text { educativa }\end{array}$ & Ordinal \\
\hline $\begin{array}{l}\text { Nivel } \\
\text { socioeconómico }\end{array}$ & $\begin{array}{l}\text { Estratificación Social basada en las remuneraciones que } \\
\text { reciben o los impuestos que pagan las personas }\end{array}$ & Ordinal \\
\hline Régimen de salud & $\begin{array}{l}\text { Vinculación de la persona al Sistema General de Seguri- } \\
\text { dad Social en Salud }\end{array}$ & Nominal \\
\hline Etnia & $\begin{array}{l}\text { Conjunto de personas que comparten rasgos culturales, } \\
\text { lengua, religión, festividades, vestimenta, tipo de alimen- } \\
\text { tación, una historia, y comúnmente un territorio. }\end{array}$ & Nominal \\
\hline Estado civil & $\begin{array}{l}\text { Su situación jurídica en la familia y la sociedad, determi- } \\
\text { na su capacidad para ejercer ciertos derechos y contraer } \\
\text { ciertas obligaciones }\end{array}$ & Nominal \\
\hline Estado laboral & $\begin{array}{l}\text { Vínculo entre una persona natural o jurídica a través de } \\
\text { un contrato escrito o verbal }\end{array}$ & Nominal \\
\hline
\end{tabular}




\begin{tabular}{lll}
\hline $\begin{array}{l}\text { Edad gestacional al ini- } \\
\text { cio de los controles pre- } \\
\text { natales }\end{array}$ & $\begin{array}{l}\text { Edad gestacional en semanas al inicio de los controles } \\
\text { prenatales. El inicio tardío de los mismos se presenta a } \\
\text { partir del segundo trimestre. }\end{array}$ & Ordinal \\
\hline $\begin{array}{l}\text { Antecedentes obstétri- } \\
\text { cos }\end{array}$ & $\begin{array}{l}\text { Historial de la mujer acerca de sus embarazos y la culmi- } \\
\text { nación de los mismos. }\end{array}$ & Nominal \\
\hline Periodo intergenésico & $\begin{array}{l}\text { Periodo de tiempo entre el último parto y el inicio de la } \\
\text { gestación actual }\end{array}$ & Ordinal \\
\hline Antecedente de IVU & $\begin{array}{l}\text { Presencia de infecciones de vías urinarias en cualquier } \\
\text { momento de la vida }\end{array}$ & Nominal \\
\hline $\begin{array}{l}\text { Asistencia a controles } \\
\text { prenatales }\end{array}$ & $\begin{array}{l}\text { Número de controles prenatales asistido durante la ges- } \\
\text { tación }\end{array}$ & Ordinal \\
\hline $\begin{array}{l}\text { Método de planificación } \\
\text { familiar }\end{array}$ & $\begin{array}{l}\text { Elemento y/o producto utilizado por la mujer para impe- } \\
\text { dir o reducir la posibilidad de embarazo }\end{array}$ & Nominal \\
\hline
\end{tabular}

El estudio tuvo aprobación del Comité de Ética en investigación de la Facultad de Salud de la Universidad Surcolombiana mediante acta número 02 del 26 de febrero de 2013.

La información se procesó en el programa Excel Microsoft office 11. Se realizó análisis univariado y bivariado mediante el programa estadístico Stata/ SE 11.0. Se utilizó como estimador epidemiológico el odds ratio pareado (OR); las pruebas de significancia utilizadas fueron el test de MacNemar chi ${ }^{2}$ con confiabilidad del 95\%. Para el ajuste de los OR pareados, se realizó regresión logística condicionada.

La confusión se controló con el pareamiento de casos y controles por edad, la aleatorización en la selección de los sujetos de estudio y el modelamiento matemático.

El sesgo de información y de memoria se evitó tomando varias fuentes de información tales como la historia clínica archivada en medio físico, magnético y el reporte del SISBEN. El sesgo de mala clasificación se evitó con la depuración realizada a la base de datos antes de la selección de casos y controles teniendo como soporte la historia clínica.

\section{Resultados}

En la presente investigación se obtuvo una muestra de 90 pacientes gestantes de las cuales se recolectó información sociodemográfica y clínica, la mediana de las edades fue de 23 años predominando las edades entre 14 y 30 años (83\%).

E1 $93 \%$ se encuentran afiliadas al sistema general de seguridad social en salud y las Empresas Promotoras de Salud más frecuentes fueron: Comfamiliar (39\%), Cafesalud Subsidiado (24\%) y Asmet Salud (13\%).

El lugar de procedencia de la muestra es en su mayoría urbano (60\%). La ocupación que predomina es la de ama de casa con un $80 \%$, el 6,6\% tienen empleo o son trabajadoras independientes y el $50 \%$ de la muestra no culminaron sus estudios de primaria y/o bachillerato.

En cuanto al estado civil y nivel socioeconómico, el 57\% estaban en unión libre durante el tiempo establecido de la investigación, el 26\% estaban solteras y el $14.4 \%$ casadas. El $54.4 \%$ de las gestantes no planificaban antes del embarazo actual y las que planificaban usaban preferentemente metodo inyectable (26\%). La etnia predominante fue mestiza (53.3\%) y el nivel socioeconómico con más frecuencia fue el nivel 1 (89\%) (Tabla 2). 
Tabla 2. Variables socioeconómicas de las gestantes participantes en el estudio, que ingresaron al control prenatal entre enero y junio de 2012 a la ESE Municipal Manuel Castro Tovar.

\begin{tabular}{|c|c|c|}
\hline Variable & Frecuencia absoluta & Frecuencia relativa $(\%)$ \\
\hline \multicolumn{3}{|l|}{ Edad (años) } \\
\hline $14-22$ & 37 & 41,1 \\
\hline $23-30$ & 38 & 42,2 \\
\hline $31-38$ & 15 & 16,6 \\
\hline TOTAL & 90 & 100 \\
\hline \multicolumn{3}{|c|}{ Régimen de seguridad social en salud } \\
\hline Contributivo & 4 & 4,4 \\
\hline Subsidiado & 84 & 93,3 \\
\hline Pobre no asegurado & 12 & 2,2 \\
\hline TOTAL & 90 & 100 \\
\hline \multicolumn{3}{|l|}{ Lugar de residencia } \\
\hline Urbano & 54 & 60 \\
\hline Rural & 36 & 40 \\
\hline TOTAL & 90 & $\begin{array}{l}40 \\
100\end{array}$ \\
\hline \multicolumn{3}{|l|}{ Ocupación } \\
\hline Ama de casa & 72 & 80 \\
\hline Empleada & 7 & 7,7 \\
\hline Trabajadora independiente & 6 & 6,6 \\
\hline Estudiante & 5 & 5,6 \\
\hline TOTAL & 90 & 100 \\
\hline \multicolumn{3}{|l|}{ Escolaridad } \\
\hline Analfabeta & 1 & 1,1 \\
\hline Primaria completa & 13 & 14,4 \\
\hline Primaria incompleta & 13 & 14,4 \\
\hline Secundaria incompleta & 32 & 35,5 \\
\hline Secundaria completa & 29 & 32,2 \\
\hline Técnico & 1 & 1,1 \\
\hline Universitario & 1 & 1,1 \\
\hline TOTAL & 90 & 100 \\
\hline \multicolumn{3}{|l|}{ Estado civil } \\
\hline Soltera & 24 & 26,6 \\
\hline Casada & 13 & 14,4 \\
\hline Unión libre & 52 & 57,7 \\
\hline Sin dato & 1 & 1,1 \\
\hline TOTAL & 90 & 100 \\
\hline \multicolumn{3}{|l|}{ Etnia } \\
\hline Mestizo & 48 & 53,3 \\
\hline Blanco & 4 & 4,4 \\
\hline Otro & 38 & 42,2 \\
\hline TOTAL & 90 & 100 \\
\hline \multicolumn{3}{|l|}{ Nivel socioeconómico } \\
\hline 1 & 73 & 88,8 \\
\hline 2 & 9 & \\
\hline 3 & 1 & 1,11 \\
\hline Sin dato & 7 & 7,7 \\
\hline TOTAL & 90 & 100 \\
\hline
\end{tabular}


En los antecedentes de IVU, el 39\% de las gestantes habian tenido alguna vez una infección de via urinaria tratada medicamente.

Los antecedentes obstetricos mostraron que la mayoría de las gestantes presentaban menos de 3 embarazos (81\%) y el 33\% eran primigestantes, la relacion entre parto/cesarea es de 4.6 , el $50 \%$ de las gestantes iniciaron control prenatal durante el primer trimestre del embarazo, y el 13\% cumplían con la mayoría de los controles prenatales, en el $47 \%$ el periodo intergenésico era de más de 2 años.

Al realizar análisis bivariado, en cuanto a antecedentes de IVU, área de residencia rural y ocupación, se demuestra que no hay asociación estadísticamente significativa entre estos factores y la presencia de IVU durante el embarazo, (OR
0,9, IC 95\% 0,3-2.3), (OR 0.75 IC95\% 0,27-1,93), (OR 0.85, IC 95\% 0.24 - 3) respectivamente. Igualmente se demuestra que la oportunidad de presentar IVU durante el embarazo es mayor para las mujeres con bajo nivel educativo $(1,33$ veces), mujeres multíparas (1,67 veces), aquellas con inicio de controles tardío (1,7 veces) y aquellas que no planificaban antes del embarazo (1,9 veces) comparado con los controles, aunque estas diferencias no son estadísticamente significativas (Tabla 3).

Finalmente se realizó un análisis multivariado con los factores propuestos en la hipótesis según el modelo de regresión logística condicionada para lo cual no se obtuvo asociación estadística entre IVU en la gestación y dichos factores (Tabla 4).

Tabla 3. Modelo bivariado de los factores antecedentes de IVU, lugar de residencia, estado laboral, escolaridad, antecedentes de embarazos, paridad, inicio de controles prenatales y planificación familiar, asociados a la infección de vías urinarias en la gestación.

\begin{tabular}{lcccc}
\hline \multicolumn{1}{c}{ Variable } & Casos & Controles & OR & IC 95\% \\
\hline $\begin{array}{l}\text { Antecedentes de IVU } \\
\text { Si ha presentado IVU alguna vez en la vida }\end{array}$ & 17 & 18 & 0,9 & $0,34-2,35$ \\
\hline $\begin{array}{l}\text { Lugar de residencia } \\
\text { Rural }\end{array}$ & 17 & 20 & 0,75 & $0,27-1,93$ \\
\hline $\begin{array}{l}\text { Estado laboral } \\
\text { Ama de casa o estudiante }\end{array}$ & 38 & 39 & 0,85 & $0,23-2,97$ \\
\hline $\begin{array}{l}\text { Escolaridad } \\
\text { Secundaria incompleta o menos nivel educativo }\end{array}$ & 31 & 28 & 1,33 & $0,51-3,58$ \\
\hline $\begin{array}{l}\text { Antecedentes de embarazos } \\
\text { Multigesta }\end{array}$ & 32 & 28 & 1,8 & $0,54-6,83$ \\
\hline $\begin{array}{l}\text { Paridad } \\
\text { Multípara }\end{array}$ & 25 & 21 & 1,66 & $0,54-5,5$ \\
\hline $\begin{array}{l}\text { Inicio de controles prenatales } \\
\text { Inicio después de la semana 12 de gestación }\end{array}$ & 23 & 18 & 1,71 & $0,62-5,13$ \\
\hline $\begin{array}{l}\text { Planificación familiar } \\
\text { No planificaba antes del embarazo }\end{array}$ & 25 & 24 & 1,1 & $0,44-2,72$ \\
\hline
\end{tabular}


Tabla 4. Relación IVU en el embarazo con escolaridad, antecedente de IVU y Paridad

\begin{tabular}{lccccc}
\hline \multicolumn{1}{c}{ Variable } & OR & Error Estandar & Z & P & IC 95\% \\
\hline Escolaridad & 1,82 & 0,91 & 1,19 & 0,23 & $0,67-4,89$ \\
\hline Antecedentes de IVU & 0,8 & 0,40 & 0,43 & 0,66 & $0,30-2,15$ \\
\hline Paridad & 1,75 & 0,96 & 1,02 & 0,31 & $0,59-5,17$ \\
\hline
\end{tabular}

\section{Discusión}

En nuestro estudio con respecto a los factores de riesgo asociados al evento, se encontró relación epidemiológica entre la presencia de IVU durante el embarazo, el bajo nivel educativo, y la paridad (no significativo por el tamaño de la muestra), como se evidencia en estudios descriptivos realizados en el Hospital Universitario de Zagazig, Egipto(17), donde se estableció asociación del evento con el bajo nivel educativo y en el Hospital Universitario de Isra, Pakistán con el evento paridad ${ }^{(18)}$. La variable "Antecedente de IVU" según lo establece la Dra Gilda Lorena Álvarez, en el estudio realizado en Argentina es un factor de riesgo para IVU durante la gestación lo que difiere con el presente estudio. Igualmente no se encontró asociación entre IVU durante la gestación, lugar de residencia rural y ocupación.

La asociación no estadísticamente significativa del antecedente de planificación familiar con la presencia de IVU durante la gestacion, sugiere la necesidad de realizar otros estudios acerca del método de planificación familiar que está predisponiendo a la aparición de dicha enfermedad. Igualmente, la asociación entre la IVU durante la gestación y la variable captación tardía de las gestantes se explica por el efecto que conlleva la adecuada educación que se brinda a las gestantes evitando eventos infecciosos durante este periodo. Igualmente estos hallazgos evidencia la falta de cumplimiento de la norma técnica de detección temprana de alteraciones del embarazo en la cual se define como actividad la captación de la gestante en las primeras
12 semanas de gestación para detectar, educar, intervenir y controlar los factores de riesgo(15).

Con respecto a la edad, se emparejó durante la selección de casos y controles, debido a que se discute sobre la influencia de esta variable en la presencia de la IVU durante la gestación. Lo anterior sustentado en los diferentes hallazgos con respecto a la variable "edad" en los estudios realizado por Álvarez (Establece asociación entre la mayoría de edad y la IVU durante la gestación) (9), Sánchez (Indica que no se logró establecer relación entre las dos variables) ${ }^{(12)}$; y Vallejo (Identifica como grupo más vulnerables las gestantes entre las edades 20 a 24 años) ${ }^{(20)}$.

No fue posible incluir en el presente estudio para el análisis de asociación la variable "bajo nivel socioeconómico" como factor asociado a la presencia de IVU en las gestantes, descrito en varios estudios ${ }^{(9,11,19)}$, puesto que el $7,7 \%$ de la muestra no tenían el dato en la historia clínica, al igual que las variables hábitos higiénicos y vida sexual activa.

Como limitaciones del presente estudio encontramos que la regular calidad de las historias clínicas, conllevó a una reducción del tamaño de la muestra (lo cual repercutió en la significancia estadística). Lo anterior aunado al tipo de diseño metodológico de la presente investigación (casos y controles) no permite realizar inferencia de los resultados a otra población, ya que la muestra al ser tomada de base hospitalaria ${ }^{(21)}$ afecta sus características de representatividad, simultaneidad y homogeneidad influyendo sobre la estimación del riesgo relativo verdadero ${ }^{(21)}$. 


\section{Conclusiones}

La oportunidad de presentar IVU en el embarazo, es mayor en las mujeres de bajo nivel educativo, multíparas, con inicio tardío de controles prenatales (después de las 12 semanas de gestación) y quienes no planificaban antes de su gestación aunque esta evidencia no es estadísticamente significativa. Tampoco se encontró asociación estadísticamente significativa entre los factores: antecedente de IVU, área de residencia, ocupación y presencia de IVU en la gestación debido a la limitación del tamaño muestral.

Dada la información socioeconómica que se recopiló en la investigación se evidencia la necesidad de estudiar prácticas sociales de las pacientes y no solo aspectos biológicos en el seguimiento de la gestación, de igual manera es importante la asistencia oportuna al control prenatal consecuente con las políticas nacionales.

\section{Agradecimientos}

Los autores expresan sus agradecimientos a la Dra. Alexandra Porras por el soporte en el desarrollo de esta investigación. Al programa de Epidemiologia de la Universidad Surcolombiana y funcionarios de la ESE Manuel Castro Tovar.

\section{Bibliografía}

1. HEILBERG I, SCHOR N. Abordagem diagnóstica e terapêutican a infecção do trato urinário: ITU.Rev. Assoc. Med. Bras.2003, vol.49, n.1, pp. 109-116. ISSN 0104-4230. (on line) http:// www.ncbi.nlm.nih.gov/pubmed/12724822 citado en 30 de septiembre de 2012

2. PASTORE, LM.; SAVITZ, DA y THORP JM Jr. Predictors of urinary tract infection at the first prenatal visit. Epidemiology 1999; 10:282-7.

3. GONZALES, Adelina. Embarazo e infección de vías urinarias. Dirección general adjunta de salud materna y perinatal. Junio 2011. Consultado de la pag: http://saludmaternamedicos.blogs- pot.com/2011/06/embarazo-e-infeccion-de-viasurinarias.html

4. JEFFREY P., Weiss, “Urologic Issues During Pregnancy," The Scientific World JOURNAL, vol. 4, pp. 364-376, 2004. doi:10.1100/tsw.2004.92. (on line) http://www.tswj.com/2004/207150/abs/

5. JIMENEZ LOPEZ, Gistet; CALVO BARBADO, Dulce y colaboradores. Terapéutica de enfermedades frecuentes en el embarazo y puerperio. Boletín de Información terapéutica para la atención primaria en salud (APS) No.30. CUBA. Julio, 2011. ISSN: 1608-7518. pdf. Consultado en Internet: 30 de Septiembre de 2012. http://files. sld.cu/cdfc/files/2011/11/boletinaps30septiembre2011.pdf

6. DUARTE G, MARCOLIN A, QUINTANA S, CAVALLI, R. Infecçãourinárian a gravidez. Rev. Bras. Ginecol.Obstet. [Online].2008, vol.30, n.2, pp. 93-100.ISSN 0100-7203. http://dx.doi. org/10.1590/S0100-72032008000200008. [citado el 30/19/2012].

7. POLANIA POLANIA, Luís Eduardo; ROJAS ROJAS, Hugo Hernando y SÁNCHEZ POLANIA, Sandra Liliana. Incidencia de patologías maternas y del recién nacido relacionadas con parto pretermino atendidos en el hospital departamental de Neiva. Universidad Surcolombiana.1998.

8. SU SB, WANG JN, LU CW, WANG HY, GUO HR. Prevalence of urinary tract infections and associated factors among pregnant workers in the electronics industry. Department of Environmental and Occupational Health, College of Medicine, National Cheng Kung University, 138 Sheng-Li Road, Tainan 70428, Taiwan.<http:// www.ncbi.nlm.nih.gov/pubmed/19415153> [24 de Junio de 2006].

9. ÁlVAREZ G, ECHEVERRÍA JC, GARAU AA, Lens VA. Argentina. Infección urinaria y embarazo. Diagnóstico y terapéutica. Argentina. Consultado en: http://med.unne.edu.ar/revista/revista155/6_155.html.

10. FIERRO, Nelson; TOVAR, Oswaldo, VERGARA Oscar. Factores de riesgo relacionados al parto pretermino al parto pretermino en mujeres que consultaron al departamento de ginecoobtetricia del hospital departamental de Neiva Hernando Moncalenao Perdomo.1996-1997. 
11. ORTIZ CA, SUÁREZ AL, GONZÁLEZ CA. Comportamiento, características clínicas y epidemiológicas de la infección de vías urinarias en mujeres gestantes ESE Laura Perdomo de García. Facultad de salud. Universidad Surcolombiana. Neiva - Huila. 2001.

12. SÁNCHEZ BC, RODRÍGUEZ, ME, RIVAS, KB et al. Factores de riesgos para Infecciones Urinarias Bajas en Embarazadas Mayo 1999-Marzo 2000. RFM, ene. 2004, vol.27, no.1, p.42-45. ISSN 0798-0469.

13. R. ABENGOZAR y otros. Problemas de salud en el embarazo. Infecciones de vías urinarias en el embarazo. Océano, 2010 p 120-125

14. Inversión_en_primera_infancia_web_(set2010). pdf (objeto application/pdf) [Internet]. [citado 2012 sep 30]. Disponible en: http://www.unicef. org/uruguay/spanish/inversion_en_primera_ infancia_web_(set2010).pdf

15. Política nacional de salud sexual y reproductiva. pdf. [Internet]. [citado 2012 sep. 30]. Disponible en: http://www.minsalud.gov.co/Documentos/ polnacionaldesaludsexualyreproductiva.pdf

16. FOCUS on Field. Epidemiology. Estudios de casos y controles. North Carolina Center for Public Health Preparedness-The North Carolina Institute for Public Health. Volumen 3. Nume ro 2.pdf
17. DIMETRY SR, EL-TOKHY HM, ABDO NM, Ebrahim MA, Eissa M. Urinary tract infection and adverse outcome of pregnancy. Community Medicine Department, Zagazig. Faculty of Medicine, Egypt. Publicado en: J Egypt Public Health Assoc. 2007; 82(3-4):203-18.

18. GULFAREEN H,NISHAT Z, AftabAfroze $r$, Ambreen Haider. Risk factors of urinary tract infection in pregnancy. Department of Obstetrics \& Gynaecology,1-3 Department of Cardiology,4 Liaquat University Hospital, Hyderabad Sindh, Pakistan. J PakMedAssoc. 2010 Mar; 60(3):213-6.

19. Guía para la detección temprana temprana de las alteraciones del embarazo.pdf. [Internet]. [citado 2012 sep 30]. Disponible en: http://www. nacer.udea.edu.co/pdf/libros/guiamps/guias03. pdf

20. VALLEJO CM, LÓPEZ MR, ENRÍQUEZ MA y RAMÍREZ B. Prevalencia de infecciones de vías urinarias en embarazadas atendidas en el Hospital Universitario de Puebla. Enfermedades Infecciosas y Microbiología, vol. 30, núm. 4, octubre-diciembre 2010.

21. LAZCANO E., SALAZAR E., Estudios epidemiológicos de casos y controles. Fundamento teórico, variantes y aplicaciones. salud pública de México / vol.43, no.2, marzo-abril de 2000, p.136. 